\title{
Common Fixed Points for JH-Operators and Occasionally Weakly g-Biased Pairs under Relaxed Condition on Probabilistic Metric Space
}

\author{
Arvind Bhatt ${ }^{1}$ and Harish Chandra ${ }^{2}$ \\ ${ }^{1}$ Department of Applied Science (Mathematics), Bipin Tripathi Kumaun Institute of Technology Dwarahat (Almora), \\ Uttarakhand Technical University, Dehradun, Uttarakhand 263653, India \\ ${ }^{2}$ Department of Mathematics and DST-CIMS, Banaras Hindu University, Varanasi 221005, India
}

Correspondence should be addressed to Arvind Bhatt; arvindbhu_6june@rediffmail.com

Received 14 May 2013; Revised 2 July 2013; Accepted 3 July 2013

Academic Editor: C. Cuevas

Copyright (C) 2013 A. Bhatt and H. Chandra. This is an open access article distributed under the Creative Commons Attribution License, which permits unrestricted use, distribution, and reproduction in any medium, provided the original work is properly cited.

We obtain some fixed point theorems for JH-operators and occasionally weakly g-biased maps on a set $X$ together with the function $F: X \times X \rightarrow \Delta$ without using the triangle inequality and without using the symmetric condition. Our results extend the results of Bhatt et al. (2010).

\section{Introduction}

Fixed point theory in probabilistic metric spaces can be considered as a part of probabilistic analysis, which is a one of the emerging areas of interdisciplinary mathematical research with many diverse applications. The theory of probabilistic metric spaces was introduced by Menger [1] in connection with some measurements in Physics. Over the years, the theory has found several important applications in the investigation of physical quantities in quantum particle physics and string theory as studied by El Naschie $[2,3]$. The area of probabilistic metric spaces is also of fundamental importance in probabilistic functional analysis. The first effort in this direction was made by Sehgal [4], who, in his doctoral dissertation, initiated the study of contraction mapping theorems in probabilistic metric spaces. Since then, Sehgal and Bharucha-Reid [5] obtained a generalization of Banach Contraction Principle on a complete Menger space which is an important step in the development of fixed point theorems in Mengar space.

Sessa [6] initiated the tradition of improving commutativity in fixed point theorems by introducing the notion of weakly commuting maps in metric spaces. Jungck [7] soon enlarged this concept to compatible maps. The notion of compatible mappings in a Mengar space has been introduced by Mishra [8]. After this, Jungck [9] gave the concept of weakly compatible maps. Aamri and El Moutawakil [10] introduced the (E.A) property and thus generalized the concept of noncompatible maps. The results obtained in the metric fixed point theory by using the notion of non-compatible maps or the (E.A) property are very interesting. Al-Thagafi and Shahzad [11] defined the concept of occasionally weakly compatible mappings which is more general than the concept of weakly compatible maps. Bhatt et al. [12] have given application of occasionally weakly compatible mappings in dynamical system. Pathak and Hussain [13] defined the concept P-operators. Hussain et al. [14] gave the concept of JHoperators and occasionally weakly g-biased.

In this paper, we obtain some fixed point theorems for JHoperators and occasionally weakly biased pairs under relaxed condition on $F$. Our results extend the results of Bhatt et al. [12].

We begin with the following basic definitions of concepts relating to probabilistic metric spaces for ready reference and also for the sake of completeness.

Definition 1 (see [15]). A real valued function $f$ on the set of real numbers is called a distribution function if it is nondecreasing, left continuous with $\inf _{t \in R} f(t)=0$ and $\sup _{t \in R} f(t)=1$. 
The Heaviside function $H$ is a distribution function defined by

$$
H(t)= \begin{cases}0, & \text { if } t \leq 0, \\ 1, & \text { if } t>0 .\end{cases}
$$

Definition 2. Let $X$ be a nonempty set and let $\Delta$ denote the set of all distribution functions defined on $X . F$ is a mapping from $X \times X$ into $\Delta$ satisfying the following condition:

$$
F_{x, y}(t)=1 \Longleftrightarrow x=y,
$$

where $F: X \times X \rightarrow \Delta$ defined by $F_{x, y}(t)=H(t-d(x, y))$ for all $x, y \in X$ and $d$ is a function $d: X \times X \rightarrow[0, \infty)$ such that $d(x, y)=0$ if and only if $x=y, \forall x, y \in X$ (symmetric and triangle conditions are not required). A topology $\tau(d)$ on $X$ is given by $U \in \tau(d)$ if and only if for each $x \in U, B(x, \epsilon) \subset U$ for some $\epsilon>0$, where $B(x, \epsilon)=\{y \in X: d(x, y)<\epsilon\}$.

Definition 3 (see $[16,17])$. Let $X$ be a non-empty set. A point $x$ in $X$ is called a coincidence point of $f$ and $g$ if and only if $f x=g x$. In this case $w=f x=g x$ is called a point of coincidence of $f$ and $g$.

Let $C(f, g)$ and $P C(f, g)$ denote the sets of coincidence points and points of coincidence, respectively, of the pair $(f, g)$. For a space $(X, F)$ satisfying $(2)$ and $A \subseteq X$, the diameter of $A$ is defined by

$$
\delta(A)=\sup \left\{\min \left\{F_{x, y}(t), F_{y, x}(t)\right\}: x, y \in A\right\} .
$$

Here we extend the concept of JH-operators and occasionally weakly g-biased pairs and the space $(X, F)$ satisfying condition (2).

Definition 4. Let $X$ be a non-empty set together with the function $F: X \times X \rightarrow \Delta$ satisfying condition (2). Two selfmaps $f$ and $g$ of a space $(X, F)$ are called JH-operators if and only if there is a point $w=f x=g x$ in $P C(f, g)$ such that

$$
F_{w, x}(t) \geq \delta(P C(f, g)), \quad F_{x, w}(t) \geq \delta(P C(f, g)) .
$$

Definition 5. Let $X$ be a non-empty set together with the function $F: X \times X \rightarrow \Delta$ satisfying condition (2). Two selfmaps $f$ and $g$ of a space $(X, F)$ are called weakly g-biased, if and only $F_{g f x, g x}(t) \geq F_{f g x, f x}(t)$ whenever $f x=g x$.

Definition 6. Let $X$ be a non-empty set together with the function $F: X \times X \rightarrow \Delta$ satisfying condition (2). Two selfmaps $f$ and $g$ of a space $(X, F)$ are called occasionally weakly g-biased, if and only if there exists some $x \in X$ such that $f x=g x$ and $F_{g f x, g x}(t) \geq F_{f g x, f x}(t)$.

Example 7. Let $X=[0,+\infty)$ and $F_{x, y}(t)=H(t-d(x, y))$, where

$$
d(x, y)= \begin{cases}e^{x-y}-1, & \text { if } x \geq y, \\ e^{y-x}, & \text { otherwise. }\end{cases}
$$

Define $f, g: X \rightarrow X$ by

$$
\begin{array}{r}
f(x)=2 x, \quad g(x)=2 x^{2}, \text { if } x \neq 0, \\
f(x)=g(x)=\frac{1}{2}, \quad \text { if } x=0 .
\end{array}
$$

In this example $C(f, g)=\{0,1\}$ and $P C(f, g)=\{1 / 2,2\}$. Here $F_{g f(1), g(1)}(t)=H(t-d(g f(1), g(1)))=H(t-d(g(2)$, $2))=H(t-d(8,2))=H\left(t-e^{6}+1\right)$. For $t=e^{6}-1, H\left(t-e^{6}+1\right)=$ $H(0)=0$. Similarly $F_{f g(1), f(1)}(t)=H(t-d(f g(1), f(1)))=$ $H(t-d(f(2), 2))=H(t-d(4,2))=H\left(t-e^{2}+1\right)$. For $t=e^{6}-1$, $H\left(t-e^{2}+1\right)=H\left(e^{6}-1-e^{2}+1\right)=H\left(e^{6}-e^{2}\right)=1$. Therefore $F_{g f(1), g(1)}(t) \leq F_{f g(1), f(1)}(t)$. Now we can easily show that $F_{g f(0), g(0)}(t) \geq F_{f g(0), f(0)}(t)$. Therefore, an occasionally weakly compatible and a nontrivial weakly g-biased pair $(f, g)$ are occasionally weakly g-biased pairs, but the converse does not hold.

\section{Section II}

We note that every symmetric (semimetric) space $(X, d)[18]$ can be realized as a probabilistic semi-metric space by taking $F: X \times X \rightarrow \Delta$ defined by $F_{x, y}(t)=H(t-d(x, y))$ for all $x$, $y$ in $X$. So probabilistic semi-metric spaces provide a wider framework than that of the symmetric spaces and are better suited in many situations. In this paper we have relaxed the symmetric condition from probabilistic semimetric space. In this section, we prove some fixed point theorems for a pair of $\mathrm{JH}$-operator on space $(X, F)$ without imposing the restriction of the triangle inequality or symmetry on $F$. In this section, we also prove some fixed point theorems for a pair of Occasionally weakly biased on space $(X, F)$ without imposing the restriction of the triangle inequality and symmetry only on point of coincidence and image of point of coincidence.

Theorem 8. Let $X$ be a non-empty set together with the function $F: X \times X \rightarrow \Delta$ satisfying condition (2). Suppose $f$ and $g$ are JH-operators on $X$ satisfying the following condition:

$$
\begin{aligned}
F_{f x, f y}(t) & \\
\geq & F_{g x, g y}\left(\frac{t}{a}\right)+\min \left\{F_{f x, g x}\left(\frac{t}{b}\right), F_{f y, g y}\left(\frac{t}{b}\right)\right\} \\
& +\min \left\{F_{g x, g y}\left(\frac{t}{c}\right), F_{g x, f x}\left(\frac{t}{c}\right), F_{g y, f y}\left(\frac{t}{c}\right)\right\},
\end{aligned}
$$

for all $x, y \in X$ with $f(x) \neq f y$ and $t>0$, where $0<a<1$, $0<b<1$, and $0<c<1$. Then $f$ and $g$ have a unique common fixed point.

Proof. We claim that $f$ and $g$ have a unique point of coincidence $w=f x=g x$. If possible, suppose there is another point of coincidence $f y=g y=w_{1}$ and $w_{1} \neq w$. Then $F_{f x, f y}(t) \neq 1 \forall t>0$. So from (7) we get

$$
\begin{aligned}
F_{f x, f y}(t) & \\
\geq & F_{f x, f y}\left(\frac{t}{a}\right)+1 \\
& \quad+\min \left\{F_{f x, f y}\left(\frac{t}{c}\right), F_{f x, f x}\left(\frac{t}{c}\right), F_{f y, f y}\left(\frac{t}{c}\right)\right\} \\
= & F_{f x, f y}\left(\frac{t}{a}\right)+1+F_{f x, f y}\left(\frac{t}{c}\right)>1 .
\end{aligned}
$$


This is a contradiction, which implies that $F_{f x, f y}(t)=1$. Hence we get $f x=f y=w=z$. Therefore there exists a unique element $w$ in $X$ such that $w=f x=g x$. Thus $\delta(P C(f, g))=1$ implies that $F_{x, w}=1$, and hence $x$ is a unique common fixed point of $f$ and $g$.

Let a function $\phi$ be defined by $\phi:[0,1] \rightarrow[0,1]$ satisfying condition $\phi(q)>q$, for all $0 \leq q<1$.

Theorem 9. Let $X$ be a non-empty set together with the function $F: X \times X \rightarrow \Delta$ satisfying the condition (2). If $f$ and $g$ are occasionally weakly g-biased on $X$, suppose

$$
F_{f w, w}(t)=F_{w, f w}(t),
$$

for some point of coincidence $w$ of $\{f, g\}$ and

$$
\begin{aligned}
& F_{f x, f y}(t) \\
& \quad \geq \phi\left[\min \left\{F_{g x, g y}(t), F_{g x, f y}(t), F_{g y, f x}(t), F_{g y, f y}(t)\right\}\right],
\end{aligned}
$$

for some $x, y \in X$ and $t>0$. Then $f$ and $g$ have a unique common fixed point.

Proof. Since $f$ and $g$ are occasionally weakly biased, there exists some $u \in X$ such that $f u=g u=w$ and $F_{g f u, g u}(t) \geq$ $F_{f g u, f u}(t)$. We claim that $f u$ is the unique common fixed point of $f$ and $g$. For if $f f u \neq f u$ then from (9) and (10) we get

$$
\begin{aligned}
& F_{f f u, f u}(t) \\
& \geq \phi\left[\min \left\{F_{g f u, g u}(t), F_{g f u, f u}(t), F_{g u, f f u}(t), F_{g u, f u}(t)\right\}\right] \\
&= \phi\left[\min \left\{F_{g f u, g u}(t), F_{g f u, g u}(t), F_{f u, f f u}(t), F_{f u, f u}(t)\right\}\right] \\
& \times(\operatorname{since} f u=g u) .
\end{aligned}
$$

Because $f$ and $g$ are occasionally weakly biased, hence,

$$
\begin{aligned}
F_{f f u, f u}(t) & \\
\geq & \phi\left[\min \left\{F_{f g u, f u}(t), F_{f g u, f u}(t), F_{f u, f f u}(t), 1\right\}\right] \\
= & \phi\left[\min \left\{F_{f f u, f u}(t), F_{f f u, f u}(t), F_{f u, f f u}(t), 1\right\}\right] \\
& \times(\text { since } f u=g u),
\end{aligned}
$$

by using condition (9),

$$
\begin{aligned}
& F_{f f u, f u}(t) \\
& \quad \geq \phi\left[\min \left\{F_{f f u, f u}(t), F_{f f u, f u}(t), F_{f f u, f u}(t), 1\right\}\right], \\
& \quad=\phi\left[\min \left\{F_{f f u, f u}(t)\right\}\right] .
\end{aligned}
$$

Since $\phi:[0,1] \rightarrow[0,1]$ satisfying the condition $\phi(q)>q$, for all $0 \leq q<1$. Therefore, $F_{f f u, f u}(t)>F_{f f u, f u}(t)$ which is a contradiction. Therefore $f f u=f u=f g u$. Hence $F_{g f u, g u}$ $(t) \geq F_{f g u, f u}=1$ which further implies that $g f u=g \mathfrak{g} u=$ $f u=f f u$. Thus $f u$ is a common fixed point of $f$ and $g$.

For uniqueness, suppose that $u, v \in X$ such that $f u=$ $g u=u$ and $f v=g v=v$ and $u \neq v$. Then (10) gives

$$
\begin{aligned}
F_{u, v}(t) & =F_{f u, f v}(t) \\
& \geq \phi\left[\min \left\{F_{g u, g v}(t), F_{g u, f v}(t), F_{g v, f u}(t), F_{g v, f v}(t)\right\}\right], \\
& =\phi\left[\min \left\{F_{u, v}(t), F_{v, u}(t), 1\right\}\right], \\
& =\phi\left[\min \left\{F_{u, v}(t), F_{v, u}(t)\right\}\right] .
\end{aligned}
$$

Let $\alpha=\min \left\{F_{u, v}(t), F_{v, u}(t)\right\}>0$. Then we get $F_{u, v}(t) \geq$ $\phi(\alpha)>\alpha$. Similarly, we get $F_{v, u}(t) \geq \phi(\alpha)>\alpha$. So, $\min \left\{F_{u, v}(t), F_{v, u}(t)\right\} \geq \phi(\alpha)>\alpha$. This is a contradiction. Therefore, $u=v$. Therefore, the common fixed point of $f$ and $g$ is unique.

Example 10. Letting $X=[0,1]$ and $\phi: X \rightarrow X$ defined as

$$
\phi(q)=\frac{1+q}{2}, \quad \text { then } \phi(q)>q, 0 \leq q<1,
$$

and $F_{x, y}(t)=H(t-d(x, y))$, where

$$
d(x, y)= \begin{cases}e^{x-y}-1, & \text { if } x \geq y \\ e^{y-x}, & \text { otherwise }\end{cases}
$$

Define $f, g: X \rightarrow X$ by $f(x)=2 x$ and $g(x)=2 x^{2}$ if $x \neq 0,1$.

One has $f(x)=g(x)=1 / 2$ if $x=0$ and $f(x)=g(x)=1$ if $x=1$.

In this example we observe that $C(f, g)=\{0,1\}$, where $(f, g)$ are occasionally weakly g-biased pairs and $F_{1, f 1}(t)=F_{f 1,1}(t)$. Now for $t=e^{1 / 2}, \phi\left[\min \left\{F_{g(1 / 2), g(1)}(t)\right.\right.$, $\left.\left.F_{g(1 / 2), f(1)}(t), F_{g(1), f(1 / 2)}(t), F_{g(1), f(1)}(t)\right\}\right]<F_{f(1 / 2), f(1)}(t)$. Example 10 is the unique common fixed point of $f$ and $g$.

Corollary 11. Let $X$ be a non-empty set together with the function $F: X \times X \rightarrow \Delta$ satisfying condition (2). If $f$ and $g$ are occasionally weakly g-biased on $X$, suppose

$$
F_{f w, w}(t)=F_{w, f w}(t),
$$

whenever $w$ is point of coincidence of $\{f, g\}$ and

$$
F_{f x, f y}(t) \geq \phi\left[F_{g x, g y}(t)\right]
$$

for some $x, y \in X$ and $t>0$. Then $f$ and $g$ have a unique common fixed point.

The proof of the following theorem can be easily obtained by replacing condition (10) by condition (20), the proof of Theorem 9.

Theorem 12. Let $X$ be a non-empty set together with the function $F: X \times X \rightarrow \Delta$ satisfying condition (2). If $f$ and $g$ are occasionally weakly g-biased on $X$, Suppose

$$
F_{f w, w}(t)=F_{w, f w}(t),
$$


whenever $w$ is point of coincidence of $\{f, g\}$ and

$$
F_{f x, f y}(t)>\min \left\{F_{g x, g y}(t), F_{g x, f y}(t), F_{g y, f x}(t), F_{g y, f y}(t)\right\},
$$

for some $x, y \in X$ and $t>0$. Then $f$ and $g$ have a unique common fixed point.

\section{Section III}

In this section, we prove several fixed point theorems for four self-mappings on $(X, F)$, where $F: X \times X \rightarrow \Delta$ satisfying condition (2). We begin with the following theorem.

Theorem 13. Let $X$ be a non-empty set and $F: X \times X \rightarrow \Delta$ satisfying condition (2). Suppose that $f, g, S$, and $T$ are selfmappings of $X$ and that the pairs $\{f, S\}$ and $\{g, T\}$ are each $\mathrm{JH}$ operators on $X$. If

$$
F_{z, w}(t)=F_{w, z}(t)
$$

whenever $w$ and $z$ are points of coincidence of $\{f, S\}$ and $\{g, T\}$, respectively, and

$$
\begin{aligned}
& F_{f x, g y}(t) \\
& \quad>\min \left\{F_{S x, T y}(t), F_{S x, f x}(t), F_{T y, g y}(t), F_{S x, g y}, F_{T y, f x}(t)\right\},
\end{aligned}
$$

for each $x, y \in X$ for which $f x \neq g y$, then $f, g, S$, and $T$ have a unique common fixed point.

Proof. By hypothesis there exist points $x, y \in X$ such that $f x=S x$ and $g y=T y$. Suppose that $F_{f x, g y}(t) \neq 1$ for all $t>0$. Then from (22),

$$
\begin{gathered}
F_{f x, g y}(t) \\
>\min \left\{F_{f x, g y}(t), F_{f x, f x}(t), F_{g y, g y}(t),\right. \\
\left.F_{f x, g y}(t), F_{g y, f x}(t)\right\}, \\
>F_{f x, g y}(t) .
\end{gathered}
$$

This is a contradiction. Hence $F_{f x, g y}(t)=1$ for all $t>0$. This implies that $f x=g y$. So $f x=S x=g y=T y$. Moreover, if there is another point $z$ such that $f z=S z$, then, using (22), it follows that $f z=S z=g y=T y$ or $f x=f z$, and $w=f x=S x$ is the unique point of coincidence of $f$ and $S$.

Thus $\delta(P C(f, S))=1$. This implies that $F_{x, f x}(t)=1$, and hence $x=w$ is a unique common fixed point of $f$ and $S$. Similarly $y=z$ is a unique fixed point of $g$ and $T$. Suppose $w \neq z$. Using (21) and (22) we get

$$
F_{w, z}(t)=F_{f w, g z}(t)>\min \left\{F_{w, z}(t), F_{z, w}(t)\right\}=F_{w, z}(t) .
$$

This is a contradiction. Therefore, $w=z$ and $w$ is the unique common fixed point of $f, g, S$, and $T$.
Let the control function $\phi: R^{+} \rightarrow R^{+}$be a continuous nondecreasing function such that $\phi(2 t) \geq 2 \phi(t)$ and $\phi(1)=1$. Let a function $\psi$ be defined by $\psi:[0,1] \rightarrow[0,1]$ satisfying the condition $\psi(q)>q$, for all $0 \leq q<1$.

Theorem 14. Let $X$ be a non-empty set and $F: X \times X \rightarrow \Delta$ satisfying condition (2). Suppose that $f, g, S$, and $T$ are selfmappings of $X$ and that the pairs $\{f, S\}$ and $\{g, T\}$ are each $J H$ operators on $X$. If

$$
F_{z, w}(t)=F_{w, z}(t),
$$

whenever $w$ and $z$ are points of coincidence of $\{f, S\}$ and $\{g, T\}$, respectively, and

$$
\psi\left(F_{f x, g y}(t)\right) \geq \psi\left(M_{\phi}(x, y)\right),
$$

where,

$$
\begin{aligned}
M_{\phi}(x, y) & \\
=\min \{ & \phi\left(F_{S x, T y}(t)\right), \phi\left(F_{S x, f x}(t)\right), \phi\left(F_{g y, T y}(t)\right), \\
& \left.\frac{1}{2}\left[\phi\left(F_{S x, g y}(t)\right)+\phi\left(F_{f x, T y}(t)\right)\right]\right\},
\end{aligned}
$$

for each $x, y \in X$ for which $f x \neq g y$, then $f, g, S$, and $T$ have a unique common fixed point.

Proof. By hypothesis there exist points $x, y$ in $X$ such that $w=f x=S x$ and $z=g y=T y$. We claim that $f x=g y$. Suppose that $f x \neq g y$. Then from (25) and (26), we get

$$
\psi\left(F_{f x, g y}(t)\right) \geq \psi\left(M_{\phi}(x, y)\right)>\psi\left(F_{f x, g y}(t)\right),
$$

which is a contradiction. Therefore $\psi\left(F_{f x, g y}(t)\right)=1$, which further implies that $F_{f x, g y}(t)=1$. Hence the claim follows that is, $w=f x=g y=z$. Now from the repeated use of condition (26) we can show that $f, g$, and $S$ and $T$ have a unique common fixed point.

Define $\mathscr{G}=\left\{\phi: \phi:\left(\mathbb{R}^{+}\right)^{5} \rightarrow \mathbb{R}^{+}\right\}$such that,

if $u \in \mathbb{R}^{+}$such that $u \geq \phi(u, 1,1, u, u)$

$u \geq \phi(1, u, 1, u, u), \quad$ or $\quad u \geq \phi(1,1, u, u, u)$,

then $u=1$.

Theorem 15. Let $X$ be a non-empty set and $F: X \times X \rightarrow \Delta$ satisfying condition (2). Suppose that $f, g, S$, and $T$ are selfmappings of $X$ and that the pairs $\{f, S\}$ and $\{g, T\}$ are each $J H$ operators on $X$. If

$$
F_{z, w}(t)=F_{w, z}(t),
$$

whenever $w$ and $z$ are points of coincidence of $\{f, S\}$ and $\{g, T\}$, respectively, and

$$
\begin{aligned}
& F_{f x, g y}(t) \\
& \geq \phi\left(F_{S x, T y}(t), F_{f x, S x}(t), F_{g y, T y}(t),\right. \\
& \left.\quad F_{f x, T y}(t), F_{g y, S x}(t)\right),
\end{aligned}
$$


for all $x, y \in X$, then $f, g$, and $S$ and $T$ have a unique common fixed point.

Proof. It follows from the given assumptions that there exists a point $x \in X$ such that $f x=S x$, and there exists another point $y \in X$ for which $g y=T y$. Suppose that $f x \neq g y$. Then, from (30) we have

$$
F_{f x, g y}(t) \geq \phi\left(F_{f x, g y}(t), 0,0, F_{f x, g y}(t), F_{g y, f x}(t)\right) .
$$

Since $f x$ and $g y$ are points of coincidence of $\{f, S\}$ and $\{g, T\}$, respectively, hence, from (30) we get,

$$
F_{f x, g y}(t) \geq \phi\left(F_{f x, g y}(t), 0,0, F_{f x, g y}(t), F_{f x, g y}(t)\right) .
$$

Therefore, from $\left(g_{1}\right)$ we get $F_{f x, g y}(t)=1$. This shows that $f x=g y$. Suppose that there exists another point $z$ such that $f z=S z$. Then, using (30) one obtains $f z=S z=g y=$ $T y=f x=S x$. Hence $w=f x=f z$ is the unique point of coincidence of $f$ and $S . \delta(P C(f, S))=1$. This implies that $F_{x, f x}(t)=1$, and hence $x=w$ is a unique common fixed point of $f$ and $S$. Similarly, there exists a unique point $v \in X$ such that $v=g z=T v$. It then follows that $v=w$, and $w$ is a common fixed point of $f, g, S$, and $T$, and $w$ is unique.

\section{Application to Dynamic Programming}

Throughout in this section, we assume that $X$ and $Y$ are Banach spaces, $S \subset X$ is a state space, and $D \subset Y$ is a decision space. We denote by $B(S)$ the set of all bounded real valued functions defined on $S$.

As suggested by Bellman and Lee [19], the basic form of the functional equations arising in dynamic programming is

$$
f(x)=\text { opt }_{y} H(x, y, f(T(x, y))),
$$

where $x$ and $y$ represent the state and decision vectors, respectively; $T$ represents the transformation of the process, and $f(x)$ represents the optimal return function with initial state $x$ (here opt denotes maximum or minimum).

We now study the existence and uniqueness of a common solution of the following functional equations arising in dynamic programming:

$$
\begin{array}{ll}
\psi(x)=\sup _{y \in D} H(x, y, \psi(T(x, y))), & x \in S, \\
P(x)=\sup _{y \in D} F(x, y, P(T(x, y))), & x \in S,
\end{array}
$$

where $T: S \times D \rightarrow S, H$ and $F: S \times D \times \mathbb{R} \rightarrow \mathbb{R}$.

As an application of Corollary 11, the existence and uniqueness of a common solution of the functional equations arising in dynamic programming can be established which extends Theorem 18 [12].

Definition 16. Let $X$ be a non-empty set and $d$ a function $d$ : $X \times X \rightarrow[0, \infty)$ such that

$$
d(x, y)=0 \quad \text { iff } x=y, \forall x, y \in X .
$$

Corollary 17. Let $X$ be a non-empty set and $d: X \times X \rightarrow$ $[0, \infty)$ a function satisfying condition (35). If $f$ and $g$ are $\mathrm{JH}$ operators self-mappings of $X$ and

$$
d(f x, f y) \leq \phi\{d(g x, g y)\}, \quad \forall x, y \in X,
$$

where $\phi: \mathbb{R}^{+} \rightarrow \mathbb{R}^{+}$a nondecreasing function satisfying the condition $\phi(t)<t$ for each $t>0$, then $f$ and $g$ have a unique common fixed point.

Proof. The proof of this corollary can be easily obtained.

We now present main result of this section.

Theorem 18. Suppose that the following conditions (i), (ii), (iii), and (iv) are satisfied.

(i) $H$ and $F$ are bounded.

(ii) $|H(x, y, h(t))-H(x, y, k(t))| \leq \phi|g h(t)-g k(t)|$ for all $(x, y) \in S \times D, h, k \in B(S)$ and $t \in S$, where $\phi: \mathbb{R}^{+} \rightarrow$ $\mathbb{R}^{+}$is a nondecreasing function satisfying the condition $\phi(t)<t$ for each $t>0$ and $f$ and $g$ are defined as follows:

$$
\begin{aligned}
& f h(x)=\sup _{y \in D} H(x, y, h(T(x, y))), \quad x \in S, h \in B(S), \\
& g k(x)=\sup _{y \in D} F(x, y, k(T(x, y))), \quad x \in S, k \in B(S) .
\end{aligned}
$$

(iii) If there is a point $f u(x)=g u(x)=k(x)$ for some $u(x) \in B(s)$ implies $d(k x, u x) \leq \delta(P C(f, g))$,

then $k(x)$ is the unique common solution of (34).

Proof. For any $h, k \in B(S)$ let

$$
d(h, k)=\sup \{|h(x)-k(x)| ; x \in S\} .
$$

From conditions (i), (ii), (iii), it follows that $f$ and $g$ are selfmappings of $B(S)$.

Letting $h_{1}, h_{2}$ be any two points of $B(S), x \in S$ and $\eta$ any positive number then there exist $y_{1}, y_{2} \in D$ such that

$$
\begin{gathered}
f h_{1}(x)<H\left(x, y_{1}, h_{1}\left(x_{1}\right)\right)+\eta, \\
f h_{2}(x)<H\left(x, y_{2}, h_{2}\left(x_{2}\right)\right)+\eta, \\
f h_{1}(x) \geq H\left(x, y_{2}, h_{1}\left(x_{2}\right)\right), \\
f h_{2}(x) \geq H\left(x, y_{1}, h_{2}\left(x_{1}\right)\right) .
\end{gathered}
$$

Subtracting (42) from (39) and using (ii), we have

$$
\begin{aligned}
f h_{1} & (x)-f h_{2}(x) \\
& <H\left(x, y_{1}, h_{1}\left(x_{1}\right)\right)-H\left(x, y_{1}, h_{2}\left(x_{1}\right)\right)+\eta \\
& \leq\left|H\left(x, y_{1}, h_{1}\left(x_{1}\right)\right)-H\left(x, y_{1}, h_{2}\left(x_{1}\right)\right)\right|+\eta \\
& \leq \phi\left(\left|g h_{1}\left(x_{1}\right)-g k_{2}\left(x_{1}\right)\right|+\eta\right) \\
& \leq \phi\left(d\left(g h_{1}, g h_{2}\right)+\eta\right) .
\end{aligned}
$$


Similarly, from (40) and (41), we get

$$
f h_{1}(x)-f h_{2}(x)>\phi\left(d\left(g h_{1}, g h_{2}\right)\right)-\eta .
$$

Hence

$$
\left|f h_{1}(x)-f h_{2}(x)\right|<\phi\left(d\left(g h_{1}, g h_{2}\right)\right)+\eta .
$$

Since (42) is true for any $x \in S$ and $\eta$ any positive number,

$$
d\left(f h_{1}, f h_{2}\right) \leq \phi\left(d\left(g h_{1}, g h_{2}\right)\right) .
$$

Therefore, from Corollary $17, k(x)$ is the unique common fixed point of $f$ and $g$; that is, $k(x)$ is the unique common solution of functional equation (34).

\section{Acknowledgment}

Authors are grateful to referee for careful reading of this paper and for valuable comments.

\section{References}

[1] K. Menger, "Statistical metrices," Proceedings of the National Academy of Sciences of the United States of America, vol. 28, no. 12, pp. 535-537, 1942.

[2] M. S. El Naschie, "On the uncertainty of Cantorian geometry and the two-slit experiment," Chaos, Solitons \& Fractals, vol. 9, no. 3, pp. 517-529, 1998 .

[3] M. S. El Naschie, "On the unification of heterotic strings, M theory and $\varepsilon\left({ }^{\infty}\right)$ theory," Chaos, Solitons \& Fractals, vol. 11, no. 14, pp. 2397-2408, 2000.

[4] V. M. Sehgal, Some fixed point theorems in functional analysis and probability [Ph.D. dissertation], Wayne State University, Detroit, Mich, USA, 1966.

[5] V. M. Sehgal and A. T. Bharucha-Reid, "Fixed points of contraction mappings on probabilistic metric spaces," Mathematical Systems Theory, vol. 6, pp. 97-102, 1972.

[6] S. Sessa, "On a weak commutativity condition of mappings in fixed point considerations," Publications de l'Institut Mathématique, vol. 32(46), pp. 149-153, 1982.

[7] G. Jungck, "Compatible mappings and common fixed points," International Journal of Mathematics and Mathematical Sciences, vol. 9, no. 4, pp. 771-779, 1986.

[8] S. N. Mishra, "Common fixed points of compatible mappings in PM-spaces," Mathematica Japonica, vol. 36, no. 2, pp. 283-289, 1991.

[9] G. Jungck, "Common fixed points for noncontinuous nonself maps on nonmetric spaces," Far East Journal of Mathematical Sciences, vol. 4, no. 2, pp. 199-215, 1996.

[10] M. Aamri and D. El Moutawakil, "Some new common fixed point theorems under strict contractive conditions," Journal of Mathematical Analysis and Applications, vol. 270, no. 1, pp. 181188, 2002.

[11] M. A. Al-Thagafi and N. Shahzad, "Generalized I-nonexpansive selfmaps and invariant approximations," Acta Mathematica Sinica, vol. 24, no. 5, pp. 867-876, 2008.

[12] A. Bhatt, H. Chandra, and D. R. Sahu, "Common fixed point theorems for occasionally weakly compatible mappings under relaxed conditions," Nonlinear Analysis: Theory, Methods \& Applications, vol. 73, no. 1, pp. 176-182, 2010.
[13] H. K. Pathak and N. Hussain, "Common fixed points for $P$ operator pair with applications," Applied Mathematics and Computation, vol. 217, no. 7, pp. 3137-3143, 2010.

[14] N. Hussain, M. A. Khamsi, and A. Latif, "Common fixed points for $J H$-operators and occasionally weakly biased pairs under relaxed conditions," Nonlinear Analysis: Theory, Methods \& Applications, vol. 74, no. 6, pp. 2133-2140, 2011.

[15] B. Schweizer and A. Skalar, Statistical Metric Spaces, North Holland, Amsterdam, The Netherlands, 1983.

[16] G. Jungck and B. E. Rhoades, "Fixed point theorems for occasionally weakly compatible mappings," Fixed Point Theory, vol. 7, no. 2, pp. 286-296, 2006.

[17] G. Jungck and N. Hussain, "Compatible maps and invariant approximations," Journal of Mathematical Analysis and Applications, vol. 325, no. 2, pp. 1003-1012, 2007.

[18] W. A. Wilson, “On semi-metric spaces," American Journal of Mathematics, vol. 53, no. 2, pp. 361-373, 1931.

[19] B. Bellman and E. S. Lee, "Functional equation arising in dynamic programming," Aequationes Mathematicae, vol. 17, pp. $1-18,1979$. 


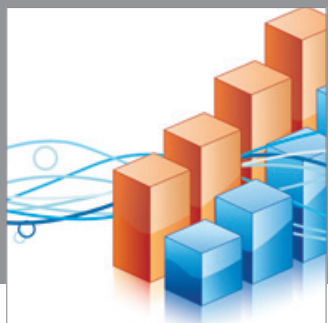

Advances in

Operations Research

mansans

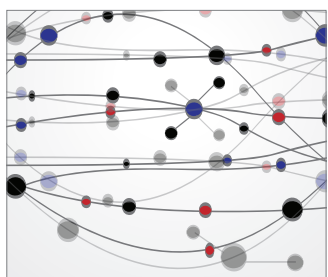

The Scientific World Journal
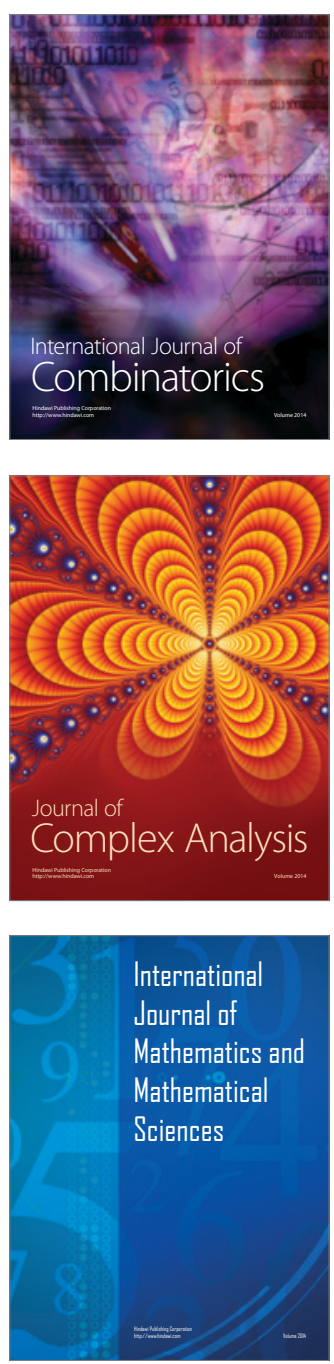
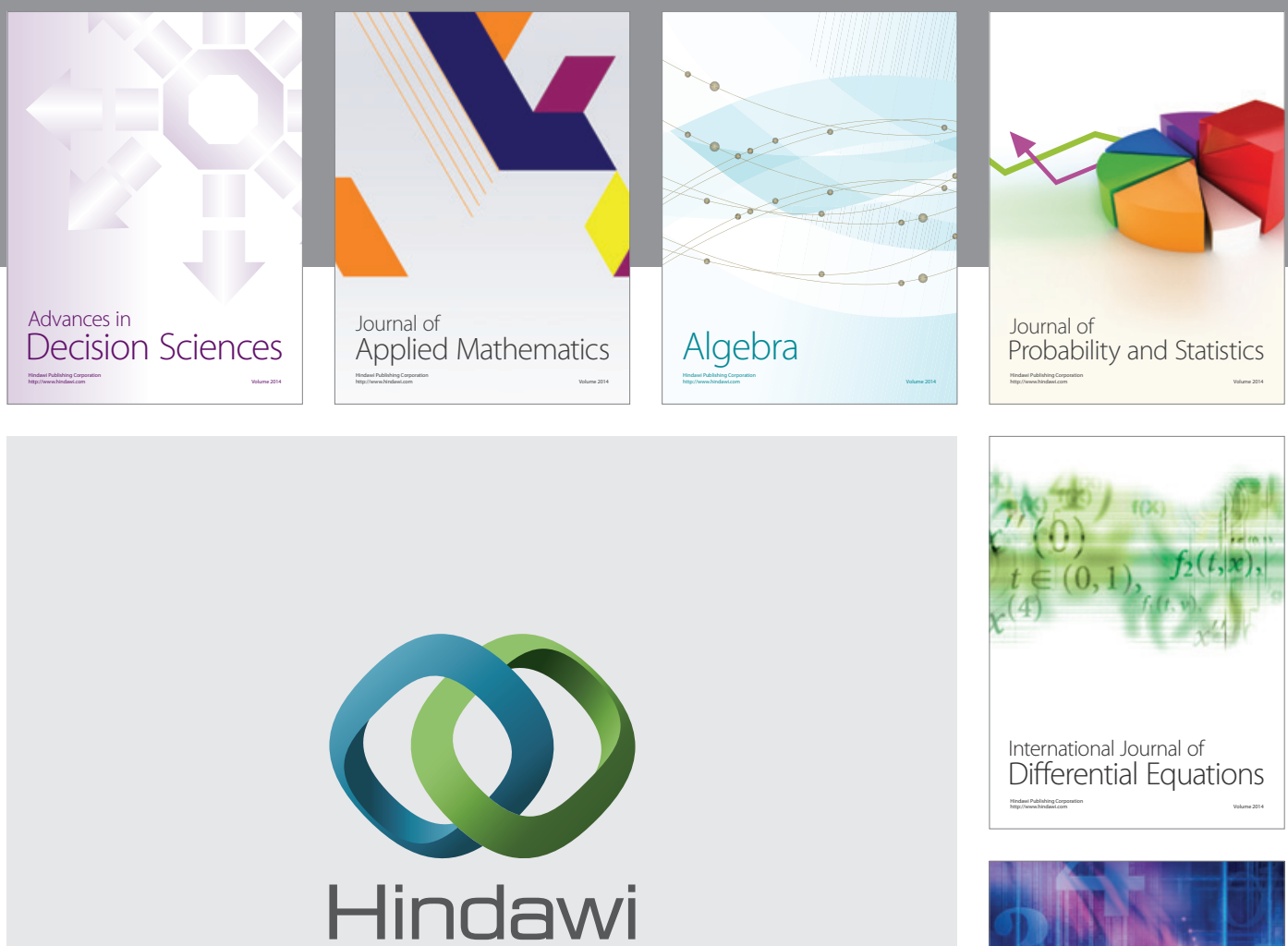

Submit your manuscripts at http://www.hindawi.com
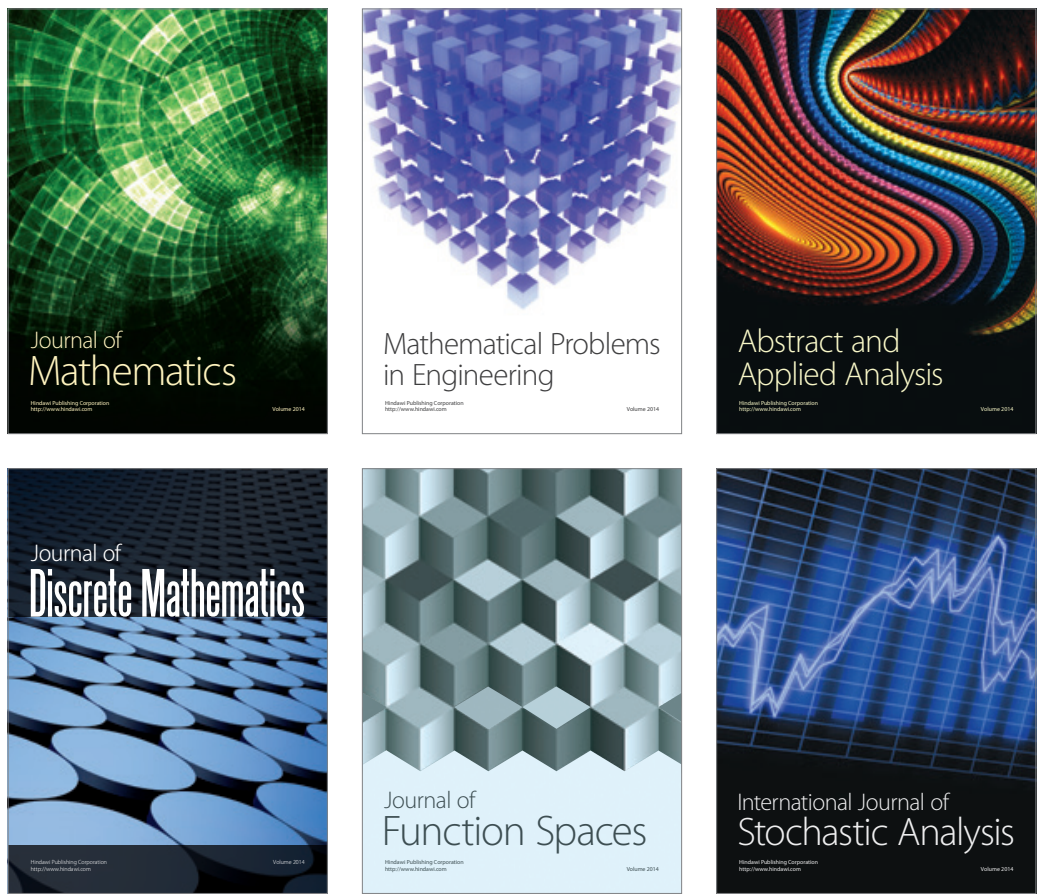

Journal of

Function Spaces

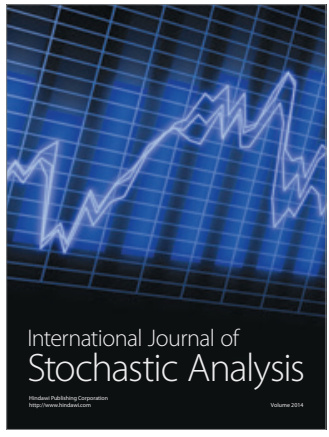

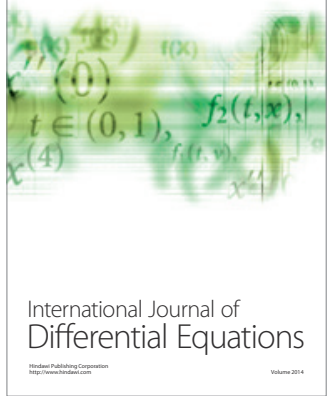
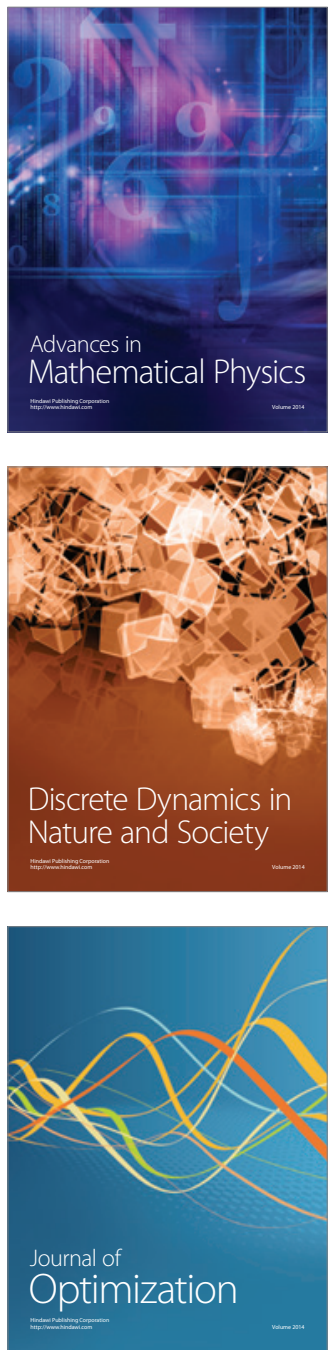